\title{
CORRECCIÓN DE ANISOMETROPÍA SECUNDARIA TRAS CIRUGÍA DE DESPRENDIMIENTO DE RETINA Y LASIK
}

\section{CORRECTION OF SECONDARY ANISOMETROPIA AFTER RETINAL DETACHMENT AND LASIK SURGERY}

\author{
DE JUAN V ${ }^{1}$, MARTÍN R ${ }^{2}$, PASTOR JC 3
}

\section{RESUMEN}

Caso clínico: Paciente intervenido hacía 7 años de LASIK que desarrolla una anisometropía cilíndrica secundaria a cirugía de desprendimiento de retina (DR) en OD. Presenta diplopia con gafas (OD $-1,25$ $\left.-2,75 \times 60^{\circ} \mathrm{AV}=1,0 ; \mathrm{OI}+0,25 \mathrm{AV}=1,2\right)$. Se adaptó una lente de contacto (LC) ajustada a su topografía corneal eliminando la diplopia.

Discusión: La cirugía del DR puede provocar cambios refractivos, induciendo anisometropía que puede dificultar su corrección con gafas. El uso de una LC adaptada a la geometría corneal disminuyó los síntomas anisometrópicos permitiendo la corrección refractiva.

Palabras clave: refracción, anisometropía, astigmatismo corneal, LASIK, indentación escleral.

\section{ABSTRACT}

Case report: A male with cylindrical anisometropia secondary to retinal detachment (RD) surgery in the right eye (OD) was referred for contact lens (CL) fitting. His refraction was OD -1.25 $2.75 \times 60^{\circ}$ VA 1.0 and $\mathrm{OS}+0.25$ VA 1.2. He was complaining of diplopia with spectacles. Seven years prior to the RD surgery, he had undergone LASIK without complications. The diplopia was eliminated after a CL was fitted according to his corneal topography.

Discussion: RD surgery can cause anisometropic refractive changes. In patients with diplopia and asthenopia, spectacles are not well tolerated. CL fitting according to post-LASIK corneal geometry succeeded in refractive correction with less anisometropic symptoms (Arch Soc Esp Oftalmol 2007; 82: 501-504).

Key words: Refraction, anisometropia, astigmatism, LASIK, scleral buckling.

\footnotetext{
Recibido: 28/9/06. Aceptado: 12/6/07.

Instituto Universitario de Oftalmobiología Aplicada (IOBA), Universidad de Valladolid, Valladolid, España.

1 Diplomado en Óptica y Optometría. Instituto Universitario de Oftalmobiología Aplicada (IOBA), Unidad de Optometría. Universidad de Valladolid.

${ }^{2}$ Diplomado en Óptica y Optometría. Instituto Universitario de Oftalmobiología Aplicada (IOBA), Unidad de Optometría. Universidad de Valladolid. Departamento de Física TAO, Universidad de Valladolid.

3 Doctor en Medicina. Instituto Universitario de Oftalmobiología Aplicada (IOBA), Grupo de Retina. Universidad de Valladolid. Servicio de Oftalmología. Hospital Clínico Universitario de Valladolid.
} 


\section{INTRODUCCIÓN}

Existen distintas técnicas quirúrgicas para el tratamiento del desprendimiento de retina (DR) regmatógeno entre las que se encuentra la cirugía de indentación escleral. Colocar un cerclaje puede provocar un cambio refractivo (1) por alargamiento de la longitud axial y/o cambios en la topografía corneal (2). Este cambio puede causar anisometropía que puede originar problemas de fusión, diplopia y otras molestias en función de su magnitud.

La anisometropía se considera clínicamente significativa cuando la diferencia de refracción entre ambos ojos es igual o superior a 1,00 dioptría (D) en uno o más meridianos. Se puede corregir con gafas, lentes de contacto (LC), cirugía refractiva o con lentes oftálmicas especiales (iseicónicas). Pero como la corrección con gafas puede inducir aniseiconia, la opción más extendida es la adaptación de lentes de contacto (3).

El tratamiento del DR en pacientes previamente intervenidos de LASIK se puede realizar con las mismas técnicas quirúrgicas que en pacientes no operados de LASIK (4) pero la corrección, óptica o quirúrgica, del posible error refractivo residual puede resultar más controvertida en función del grado de ametropía inducida, del espesor y la topografía corneal y de la tolerancia al uso de lentes de contacto (5).

Se presenta la adaptación de lentes de contacto en un paciente intervenido de LASIK con anisometropía secundaria a cirugía de DR.

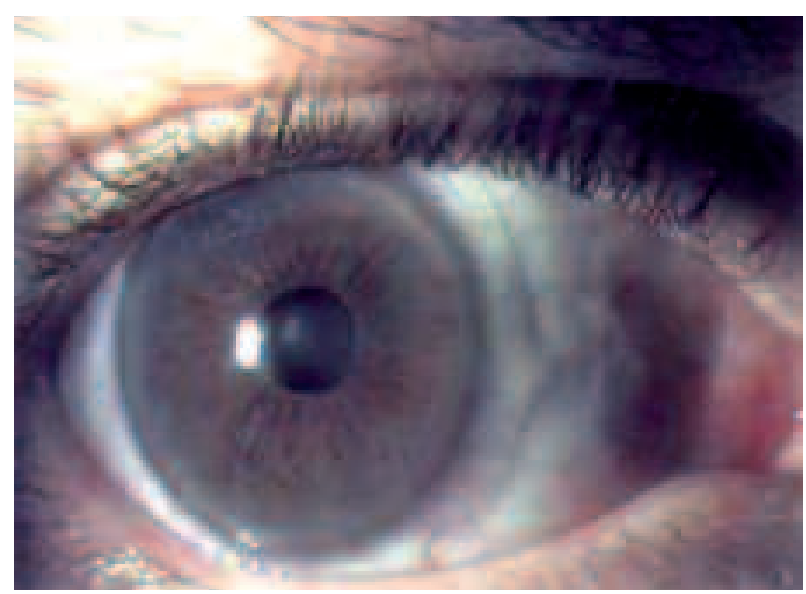

Fig. 1: Aspecto biomicroscópico de la lente de contacto con un centrado óptimo. El diámetro de 14,5 mm permite una estabilización de la lente adecuada, manteniendo el centrado y una visión estable.

\section{CASO CLÍNICO}

Varón de 46 años con metamorfopsias en su ojo derecho (OD) y refracción subjetiva de $-1,25$ $-2,25 \times 60^{\circ}$, con una $\mathrm{AV}=1,0$ y en $\mathrm{OI}=+0,25$ con $\mathrm{AV}=1,2$, que le provoca diplopía y mareo. El paciente había sido intervenido de un DR en OD tres años antes mediante vitrectomía, cerclaje y taponamiento con gas $\left(\mathrm{SF}_{6}\right)$. Cursó con reaplicación de la retina. Previamente (7 años antes) había sido operado de LASIK ( $-6,50 \mathrm{D}$ en ambos ojos) sin complicaciones y con buen resultado refractivo y visual.

Se adaptó una LC hidrofílica moldeada de bajo espesor $(0,1 \mathrm{~mm}$ para $-3,00 \mathrm{D})$ de toro interno y sistema de estabilización con prisma balastrado ajustada a su topografía corneal periférica, radio base $8,9 \mathrm{~mm}$, diámetro de $14,5 \mathrm{~mm}$ y potencia $-1,25$ $-2,50 \times 70^{\circ}$ consiguiendo AV de 1,0 (fig. 1). Durante la adaptación fue necesario corregir el eje de la lente al estabilizar con un giro de $10^{\circ}$ en sentido horario. La adaptación fue satisfactoria con buena tolerancia subjetiva desapareciendo la diplopia y los síntomas astenópicos.

\section{DISCUSIÓN}

La adaptación de LC para eliminar la diplopia por anisometropía es ampliamente conocida (disminuye la distancia al vértice minimizando la aniseiconía). Actualmente existe una amplia gama de materiales y geometrías que permiten la adaptación de LC de forma segura y eficaz en un elevado número de personas. Sin embargo, en ojos intervenidos de LASIK miópico la topografía corneal cambia de forma prolata a oblata y la adaptación de LC puede requerir el uso de diseños especiales, tratándose de una adaptación más compleja que en un ojo no intervenido, con peor tolerancia ya que uno de los principales motivos para la cirugía suele ser la intolerancia previa al uso de LC y también se ha descrito la aparición de ojo seco tras LASIK.

La refracción postquirúrgica del paciente es compatible con los cambios refractivos que puede provocar la cirugía de indentación escleral, aumentando la longitud axial del globo ocular, que induce miopía, y afectando a la curvatura corneal, apareciendo astigmatismo (4). El astigmatismo refractivo y corneal del OD eran similares (fig. 2). 


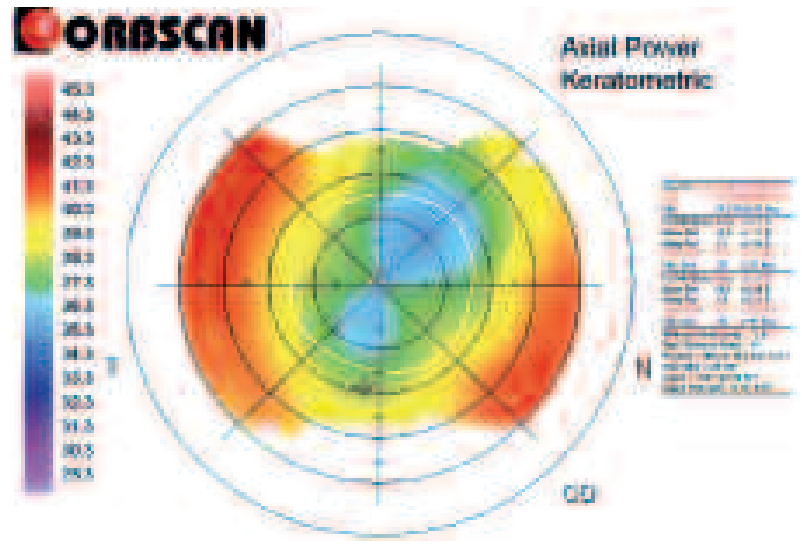

Fig. 2: Topografía corneal. Se aprecia el astigmatismo corneal regular de 2,00 D muy similar al refractivo de 2,25 D. La queratometría central era de 9,15 (50 $) x$ $8,70\left(140^{\circ}\right)$. La potencia en la periferia $(5,0 \mathrm{~mm})$ era de 39,20 D (8,6 mm) lo que permitió un ajuste adecuado con una lente de radio $8,9 \mathrm{~mm}$.

En teoría, las alternativas de corrección para la anisometropía son gafas, LC y cirugía refractiva. La corrección en gafas provocaba mareo, diplopia, etc. por lo que se descartó. La cirugía refractiva se descartó al haber sido operado de LASIK y presentar una anisometropía inducida por el cerclaje que en algunos casos debe ser retirado con lo que la anisometropía se modificaría, por lo que se propuso la corrección con LC. Después de LASIK miópico está indicada la adaptación de LC rígidas permeables a los gases (RPG) de geometría inversa, especialmente en presencia de córneas irregulares (5). Este caso presentaba un astigmatismo regular y la adaptación monocular de una lente RPG podía presentar más dificultades de tolerancia y hacer fracasar la adaptación. Además, el paciente había presentado problemas de tolerancia al uso de lentes RPG previamente. Se decidió adaptar una LC hidrofílica tórica. Para la selección de sus parámetros se buscó el mejor ajuste con la periferia corneal consiguiendo una adaptación óptima que permitía una AV de 1,0 sin síntomas de mareo o diplopia.

El ajuste a la topografía periférica corneal y la selección de una lente de fabricación moldeada con un espesor reducido puede permitir la adaptación de forma satisfactoria y cómoda en casos de anisometropías postquirúrgicas que no puedan corregirse con gafas o cirugía refractiva.

\section{BIBLIOGRAFÍA}

1. Citirik M, Batman C, Acaroglu G, Can C, Zilelioglu O, Koc F. Analysis of changes in corneal shape and bulbus geometry after retinal detachment surgery. Int Ophthalmol 2004; 25: 43-51.

2. Sinha $R$, Sharma $N$, Verma L, Pandey RM, Vajpayee RB. Corneal topographic changes following retinal surgery. BMC Ophthalmol 2004; 4: 10.

3. Taylor M, Raasch T, Polasky M. Patients with anisometropia and aniseikonia. In: Benjamin WJ Borish's clinical refraction. Philadelphia: W.B. Saunders Company; 1998; 1134-1159.

4. Arevalo JF. Retinal complications after laser-assisted in situ keratomileusis (LASIK). Curr Opin Ophthalmol 2004; 15: 184-191.

5. Martin R, Rodriguez, G. Reverse geometry contact lens fitting after corneal refractive surgery. J Refract Surg 2005; 21: 753-756. 